\title{
Simple and Efficient Blood Glucose Measurement Technique Using Non Invasive Artificial Intelligence
}

\author{
Asif Khan and Dr. Shahab Khushnood
}

\begin{abstract}
At present, roughly about 50.8 million people in the world suffering from diabetes and maintaining the blood sugar level is extremely important. Diabetic patients need to frequently check their glucose level by means of pricking the finger for taking blood samples 4-5 times a day and maintain their sugar level through the process of taking appropriate dosage of medicine. In this paper, an artificial intelligence based image processing technique has been formulated, which non-invasively computes the glucose level exist in the urine sample of a person and therefore the corresponding blood glucose level of that person is computed. Blood sugar level of a person from his/her urine sample has been examined by observing the colour transformation of the test sample, when it is reacted with Benedict's reagent. The colour transformation of the sample is recognized with the assistance of the camera and shows the result in the form of hue* value. This measurement can be effectively carried out by training the neural network with the hue value as the input vector and the glucose value as the test vector. Results show that the linear relationship has been obtained successfully with accuracy in the range of $96.93 \%$.
\end{abstract}

Keywords--- Benedict's Reagent, Colour Sensor, Neural Network, HSI Colour Format, Diabetes Mellitus.

*Note: Hue - predominant Colour

\section{INTRODUCTION}

$\mathrm{D}$ IABETES mellitus is a deficiency in which the body cannot regulate the amount of glucose (sugar) in the blood [1]. The glucose present in the body provides the energy to perform person's daily activities. The liver performs the conversion of food into glucose and the glucose is then released into the bloodstream. The blood glucose level of a healthy person is regulated by hormones like insulin. Insulin is produced by the pancreas, a small organ that lies between the stomach and liver. The pancreas also releases some other enzymes which help to digest the food. Insulin is very helpful in moving the glucose out of the blood into the cell throughout the body, where it is used as a fuel [2].

People with diabetes either do not produce enough insulin or cannot use insulin properly in day-to-day life or both. In diabetes, glucose cannot move efficiently into the cells, so glucose level in the blood remains high. This starves the cells

Asif Khan, University of Engineering and Technology, Pakistan.

Dr. Shahab Khushnood, Harvard University, USA.

DOI : 10.9756/BIJIEMS.8320 which needs the fuel (i.e) glucose. It also harms the organs and tissues exposed to high glucose levels.

There are different forms of diabetes. Type 1 diabetes is a type of diabetes in which the body cells stop producing insulin or it produces too little insulin to regulate the blood sugar level [3]. Type 1 diabetes is usually referred to as insulin-dependent diabetes. It usually occurs at the older age due to damage in the pancreas by alcohol or diseases. Type 2 diabetes is a type of diabetes in which the insulin produced by the pancreas is partially or completely not used by the blood cells [4]. It is usually referred to as insulin resistance or insulin-independent diabetes. This type of diabetes does occur at the younger age and so some people require insulin therapy. It is usually controlled with diet, weight loss, exercise and oral medicines.

The main symptoms of the diabetes are loss of weight, frequent urination, increased thirst and increased hunger [5]. The symptoms may develop rapidly in type 1 diabetes, while they usually slower or it may be absent in type 2 diabetes[6]. Prolonged hyper glycemia leads to changes in the shape of a lens present in eye, resulting in blurred vision. Type 1 diabetes can easily be identified by rapid vision change whereas type 2 diabetes is generally gradual. The skin rashes also occurs in diabetes, which is commonly known as diabetic dermadromes [7][8].

Conventionally diabetes can be diagnosed by blood or urine of a person. There are three main categories for the measurement of glucose level. Invasive technique, which involves pricking of hand to take blood samples or implanting sensors into a human body for continuous monitoring of a glucose level [9]. Semi invasive technique, which involves taking single drop of blood sample to measure the glucose value. Non-invasive technique, which measures the glucose concentration by taking urine sample instead of pricking the finger for taking the blood samples [10]. This paper mainly deals with the non-invasive technique which continuously monitors the blood glucose level by taking the urine sample of a person. This technique would not cause pain and it is a cost effective.

\section{EXPERIMENTAL SETUP}

The experimental set of the system is shown in the figure 1. In figure 1, there are three ports namely inlet port, reagent port and outlet port. The inlet port opens to collect the urine sample. The reagent port allows to collect the Benedict's reagent. The heater is used to boil the mixer up to the boiling point automatically, which is controlled by computer. The colour change of the mixer is sensed by Camera and after 
sensing the colour, the waste is removed through the outlet port. The colour sensed by the camera is then processed in personnel computer by an artificial intelligence based system. The entire process is explained with the help of a flow diagram which is shown in figure 2.

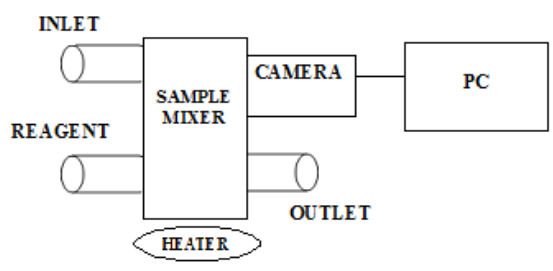

Figure 1: Experimental Setup

\section{MATERIALS AND METHODS}

The entire work is an amalgamation of mechanical and control engineering, chemistry, image processing and neural network. The next section will explain the system details.

\section{A. Benedict's Reagent}

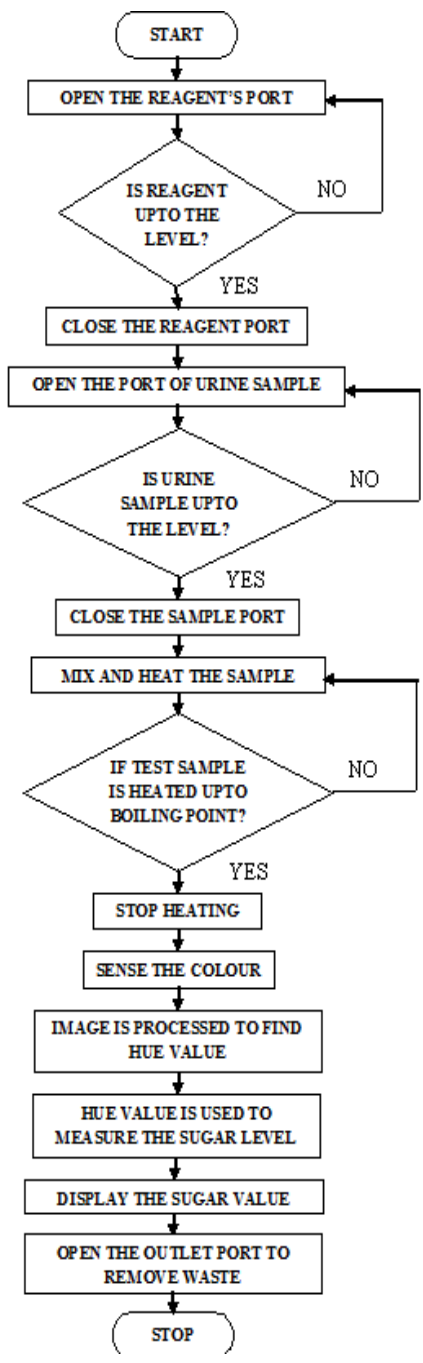

Figure 2: Flow Diagram

Benedict's solution acts as a reagent to find the blood sugar level. Benedict's solution is used to find the sugar level present in the urine which gives the equivalent blood sugar value [11]. Benedict's reagent is composed of copper sulphate, sodium carbonate and sodium citrate. When Benedict's reagent is reacted with urine sample, its colour changes based on the amount of sugar level. The healthy persons normally have the blood glucose concentration in the range of 70$120 \mathrm{mg} / \mathrm{dl}$. Formation of red, green and yellow precipitate denotes the positive result for glucose test. Blue colour denotes the NIL sugar that means blood sugar level is less than $180 \mathrm{mg} / \mathrm{dl}$. Green precipitate indicates the blood glucose level is appropriate to $180-220 \mathrm{mg} / \mathrm{dl}$. Yellow precipitate denotes the glucose concentration is appropriate to $221-280 \mathrm{~g} / \mathrm{dl}$. Red precipitate represents the blood sugar level in the range of 281-350mg/dl. Brick red colour denotes the sugar level is very high above $350 \mathrm{mg} / \mathrm{dl}$.

Figure 3 shows the different colours of the urine sample reacted with the Benedict's reagent[12]. In olden days the blood sugar value is predicted with the help of these colour changes. In this paper with the help of this reference value, an accurate blood sugar value is easily identified by capturing and processing the image obtained.

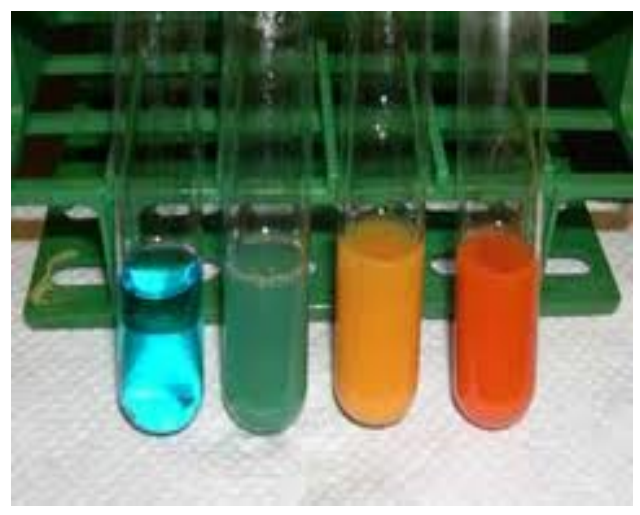

Figure 3: Different Colours of Urine Sample Reacted with the Benedict's Reagent

\section{B. System Description}

Figure 4 shows the block diagram of the system. The urine is collected in a test tube. Benedict's reagent $(5 \mathrm{ml})$ is added to the collected sample and it is mixed thoroughly to get a test sample. Holding the test tube with holder and heat it up to the boiling point with the help of a heater. Then allow the solution to cool down for few minutes, while cooling the mixture changes its colour. The colour of the mixture which serves as a guide to the amount of sugar in the urine which infer the equivalent blood sugar level[13]. The colour change is then captured by colour sensor and the image is loaded into the PC for further calculation using artificial intelligence technique. The solution is then drained out as a waste.

\section{Image Processing}

The colour change obtained after heating the mixer is captured by camera. The colour change obtained is uniform but the image captured by the colour sensor is generally noise. In order to obtain the original image, convolution based filtering is used. This type of filtering removes the noise from the image and gives the original image. 


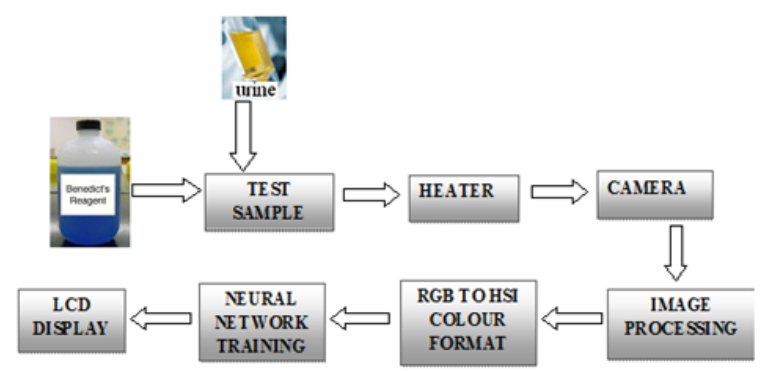

Figure 4: Block Diagram of the System

Convolution based filtering is used separately on the Red, Green and Blue components of the sensed image by equation 1

$$
g(x, y)=\sum_{u=-a v=-b}^{a} \sum^{b} w(u, v) f(x+u, y+v)
$$

where, $\mathrm{w}$ is the convolution mask of size (mxn),

$\mathrm{f}$ is the original image captured with noise,

$\mathrm{x}$ and $\mathrm{y}$ is the row and column indexes of the image.

The convolution mask of size (mxn) have the coefficient values equal to $1 /(\mathrm{mxn})$. The $\mathrm{m}$ values ranges from $-\mathrm{a}$ to $+\mathrm{a}$ and the $n$ values ranges from $-b$ to $+b$, where $a$ and $b$ are nonnegative integers. The coefficient value $\mathrm{w}(0,0)$ coincides with the image value $f(x, y)$, such that the convolution mask is centered at $(\mathrm{x}, \mathrm{y})$ position of the sensed, where the computation takes place. $\mathrm{g}(\mathrm{x}, \mathrm{y})$ finally gets the response of the filter. After applying the filter to the noisy image the red, blue and green components are assembled again to build the original image. The sensed image is shown in the figure 5.

The next step is to extract the colour information from the sensed image. The original image contains only the information about the primary colours that is RGB components. The main drawback of using RGB components is that it not only contains the colour information but also the colour information but also contains the colour intensity.

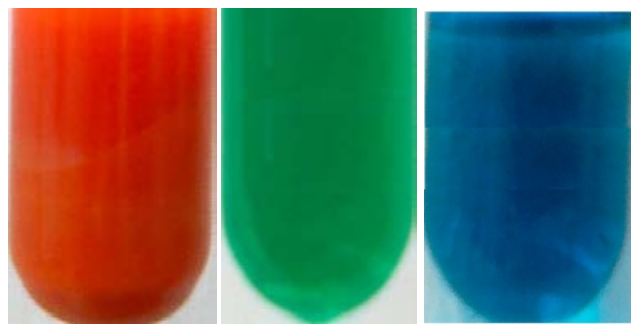

Figure 5: The Sensed Original Image

The RGB values are different for different intensities i.e it is different for light blue, dark blue and navy blue. With the RGB values the original colour of the image is not easily identified. To overcome this problem different colour format has been used. The HSI colour format is suggested to find the predominant colour of the original image, where $\mathrm{H}$ stands for Hue i.e pure colour, S stands for Saturation i.e the degree by which the pure colour is diluted by the white colour and I stands for the Intensity i.e grey level value.
The RGB to HSI colour format conversion is required to find the pure colour of an image. The HSI colour format conversion is performed using following equations.

$$
\begin{aligned}
& H=\left\{\begin{array}{l}
\theta \ldots \ldots . ., \text { if } . . B \leq G \\
360-\theta, \text { otherwise }
\end{array}\right\} \\
& \theta=\cos ^{-1}\left\{\frac{\frac{1}{2}[(R-G)+(R-B)]}{\sqrt{[(R-G)(R-G)+(R-B)(G-B)}}\right\} \\
& S=1-\frac{3}{(R+G+B)}[\min (R, G, B)] \\
& I=\frac{1}{3}(R+B+G)
\end{aligned}
$$

In the HSI components, Saturation and Intensity should have values in the range $[0,1]$ and Hue should have values in the range $[0,360]$

\section{Algorithm to find the Hue Value Matrix from the Image}

RGB stands for Red, Green and Blue components of the image. " $\mathrm{H}$ " is a matrix which contains the Hue from an image. "Row" is a vector from a hue matrix. "minimum()" is a function which returns the minimum hue value.

1. Step 1: Equation 3 is applied to each pixel of the RGB components of an image and the Hue values that are obtained are stored in the $\mathrm{H}$ matrix.

2. Step 2: The middle element is found from the $\mathrm{H}$ matrix by sorting entire row's element and store it in a vector named "Row".

3. Step 3: Sort the "Row" vector and the middle element is taken as a Hue value.

4. Step 4: As already seen the Hue value varies from 0 to 360, hence Actual Hue = minimum (Hue, 1-Hue).

5. Step 5: Return the Actual Hue value.

6. Step 6: Stop.

\section{Deciding the Sugar Level}

It is very difficult to identify the pure colour from the hue value of an image. So some other techniques should be followed to overcome this problem. Neural network training pattern is used to solve this problem. Neural network is an artificial intelligence based method, which can be viewed as a neurons processing in a human brain[14]. Neural networks are a form of multiprocessor computer systems with simple processing elements and high degree of interconnections. There are many types of neural network models available. In this paper, back propagation model is adapted to train the network pattern.

Back propagation neural network is derived from the delta rule, in which extra hidden layers can be added to show the percentage of sugar more precisely. The input and the target vectors should be fed initially for training the network pattern. Training these networks is done by changing the weights of the unit depends on the error occurring at that unit. The weight change rule is developed from the perceptron learning rule. 
The output unit error is used to alter the weights on the output unit. The hidden layer errors can be calculated by back propagating the errors at the output unit and the hidden layer weights are altered with those errors. For each data set, the forward pass and backward pass is continued until the error become too low. Unlike other networks, in back propagation network the errors can be back propagated to the hidden layers, so that more accurate results can be obtained. The structure of the back propagation neural network was shown in figure 6 .

In this project single layer net is used which means one input unit, one hidden layer and one output unit. To get the more accurate results hundreds of iterations should be done to achieve the target output. More number of iterations is to be made to correct the errors by forward pass and backward pass. The forward pass and the backward pass can be done using the following equations.

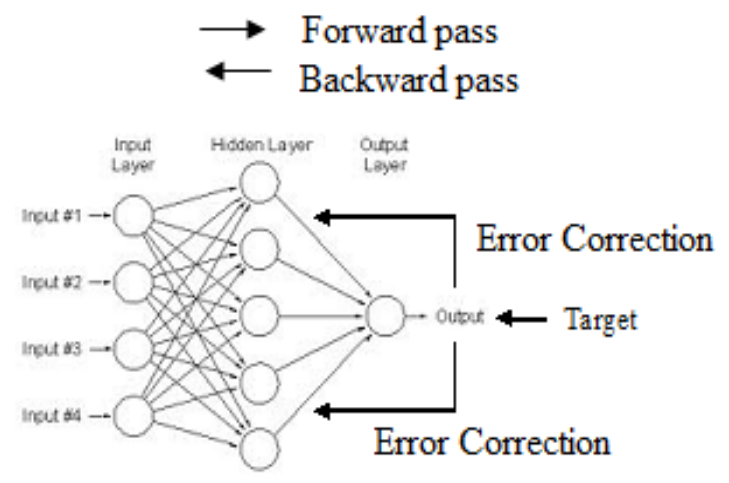

Figure 6: Back Propagation Neural Network

Table 1: Training Test Vector Sugar Measurement

\begin{tabular}{|c|c|c|c|c|c|c|c|}
\hline \multicolumn{2}{|c|}{ Red colour } & \multicolumn{2}{c|}{$\begin{array}{c}\text { Yellow } \\
\text { colour }\end{array}$} & \multicolumn{2}{c|}{$\begin{array}{c}\text { Green } \\
\text { colour }\end{array}$} & \multicolumn{2}{c|}{ Blue colour } \\
\hline Hue & $\%$ & Hue & $\%$ & Hue & $\%$ & Hue & $\%$ \\
\hline 0.007 & 100 & 0.039 & 20 & 0.184 & 10 & 0.417 & 20 \\
\hline 0.039 & 80 & 0.060 & 30 & 0.192 & 20 & 0.452 & 30 \\
\hline 0.060 & 70 & 0.082 & 40 & 0.214 & 70 & 0.481 & 60 \\
\hline 0.082 & 60 & 0.106 & 50 & 0.245 & 80 & 0.5 & 80 \\
\hline 0.106 & 50 & 0.127 & 60 & 0.272 & 100 & 0.510 & 100 \\
\hline 0.127 & 40 & 0.148 & 70 & 0.314 & 100 & 0.534 & 100 \\
\hline 0.148 & 30 & 0.166 & 90 & 0.333 & 100 & 0 & 0 \\
\hline 0.166 & 10 & 0.184 & 90 & 0.394 & 90 & 0 & 0 \\
\hline 0 & 0 & 0.192 & 80 & 0.417 & 80 & 0 & 0 \\
\hline 0 & 0 & 0.214 & 30 & 0.452 & 70 & 0 & 0 \\
\hline 0 & 0 & 0.245 & 20 & 0.481 & 40 & 0 & 0 \\
\hline 0 & 0 & 0 & 0 & 0.5 & 20 & 0 & 0 \\
\hline
\end{tabular}

The forward pass:

Input to the hidden unit,

$$
\mathrm{Z}_{\mathrm{inj}}=\mathrm{V}_{0 \mathrm{j}}+\sum_{i} x_{i} v_{i j}
$$

Output of the hidden layer,

$$
\mathrm{Z}_{\mathrm{j}}=f\left(\mathrm{Z}_{\mathrm{inj}}\right)
$$

Input to the output unit,

$$
\mathrm{Y}_{\mathrm{ink}}=\mathrm{W}_{0 \mathrm{k}}+\sum_{j=1}^{p} Z_{j} W_{j k}
$$

To compute the output signal,

$$
\mathrm{Y}_{\mathrm{k}}=f\left(\mathrm{Y}_{\text {ink }}\right)
$$

The backward pass:

The error correction term of the output unit,

$$
\delta k=\left(T_{k}-Y_{k}\right) f^{\prime}\left(Y_{\text {ink }}\right)
$$

Updating the weights and bias of the output unit,

$$
\begin{aligned}
\Delta W_{j k} & =\alpha \delta_{k} Z_{j} \\
\Delta W_{0 k} & =\alpha \delta_{k}
\end{aligned}
$$

Error correction term of the hidden unit,

$$
\begin{aligned}
& \delta_{i n j}=\sum_{k=1}^{m} \delta_{k} W_{j k} \\
& \delta_{j}=\delta_{i n j} f^{\prime}\left(\mathrm{Z}_{\text {inj }}\right)
\end{aligned}
$$

Update the weight and bias of the hidden unit,

$$
\begin{aligned}
& \Delta V_{i j}=\alpha \delta_{j} x_{i} \\
& \Delta V_{0 j}=\alpha \delta_{j}
\end{aligned}
$$

Where $\mathrm{i}$ is the number of input vectors

$\mathrm{j}$ is the number of hidden vectors

$\mathrm{k}$ is the number of output vectors

$\mathrm{T}$ is the target unit

The equation (11) and (15) update the weights to reduce the errors. In blood glucose measuring technique by urine and Benedict's reagent, four colours test set is required. The sugar level is identified with the help of the four colours like blue, green, yellow and red. By majority voting technique, the training test set is formed and the final decision is to be made by this test vector.

The next session describes the algorithm to find the percentage of sugar present in the urine with the help of Hue value obtained. The input and the target vector are initially assigned to the neural network. The weights and bias is also randomly assigned. After training the network pattern to the minimum error value, the calculation for finding the sugar value has to be done.

1. Step 1: Assign the minimum and the maximum value of the input test vector.

2. Step 2: Set product value $=1$.

3. Step 3: Output $=$ bias + (activation function * weights).

4. Step 4: Find the ratio for the de-normalization.

5. ratio $=[\max -\min$ of the input vector $] /$ [max-min of the reference values taken]

6. Step 5: De-normalization process is done. $\mathrm{Nt}=$ (output + product value)/ratio

7. Step 6: Final output $=\mathrm{Nt}+$ minimum reference value. 
The trained test vector by majority voting technique for measuring the sugar value is shown in table 1 .

\section{SimUlation AND Result}

The simulation for the artificial intelligence based image processing is done by MATLAB tool box. The simulation is used to extract the final hue value from the sensed image by image processing tool box and the extracted hue value is trained by the neural network toolbox to get the blood sugar value.

Figure 7 shows the graph of hue value of the test sample Vs percentage of sugar level of each colour image. The red vertical line shows the sugar level of red colour image which indicates the maximum glucose content. Yellow line shows the response of the yellow colour image which denotes the sugar value in the range of 221-280 mg/dl. Similarly Green and the Red line shows the response of the corresponding image.

The algorithm used in this paper gives the more precise hue value. The hue value obtained for red colour image in figure 5 is 0.0277 which is shown in figure 8. This pure value can be used to find the percentage of sugar value that is being present in the red image. The final output value for red colour image is 88.1713 which indicates the maximum amount of glucose in the blood is shoen in figure 9. So, the final decision is that the blood sugar value is much higher for that particular patient and the appropriate dosage of medicine should be given.

For testing purpose nearly 200 samples are used in which 194 samples gives the satisfactory results. Thus the error rate is up to $3.07 \%$.

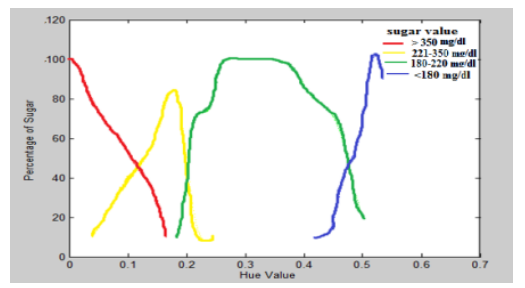

Figure 7: Hue Value Vs Percentage of Sugar

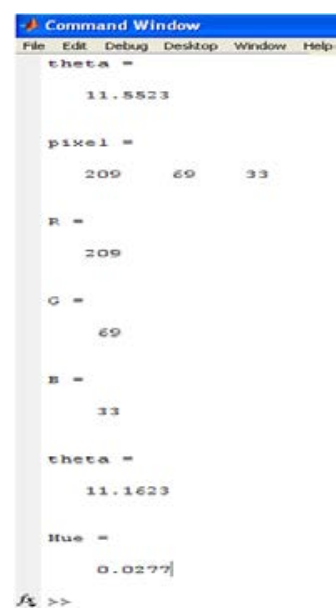

Figure 8: Hue Value for the Red Colour Image

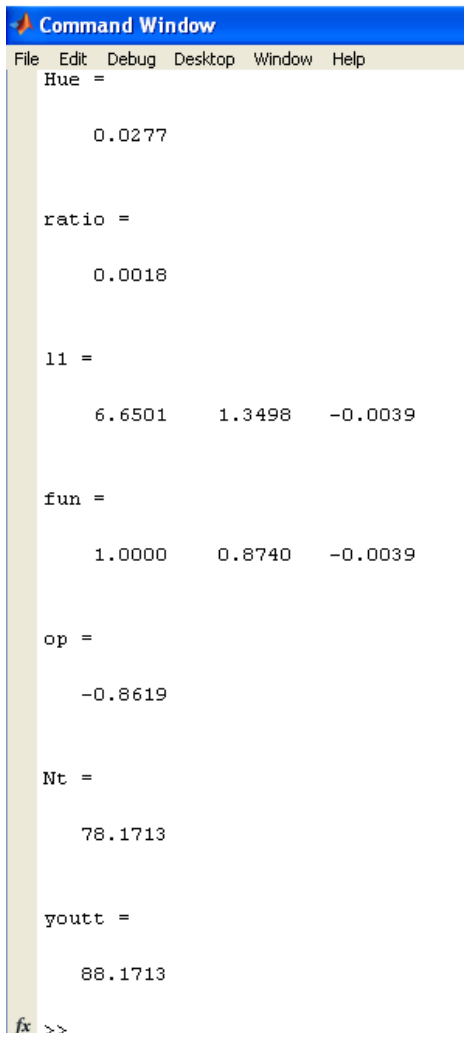

Figure 9: Percentage of Sugar Value for Red Colour Image

\section{CONCLUSION AND DisCUSSION}

This system is very simple and efficient to detect the blood sugar level and also cost effective with no pain. It can reduce the probability of glaucoma, kidney problem, blurred vision, etc by early detection of high blood glucose level. The algorithm used for this approach is very simpler and provides the accuracy of about $96.93 \%$. Future work of this system is to enhance the decision algorithms containing large training sets, so that the result will be more accurate.

\section{REFERENCES}

[1] R.C. Bateman and J.A. Evans, "Using the glucose oxidase/peroxidase system in enzyme kinetics”, J. Chem. Educ., Vol. 72, No. 12, Pp. A240-A241, 1995.

[2] Ragnar Hanas, "Type 1 diabetes in children, adolescents and young adults”, 3th ed. Uddevalla, Sweden, 2007.

[3] P. English and G. Williams, “Type 2 Diabetes”, Taylor \& Francis, 2001.

[4] C. Brooker, "Human structure and function: nursing applications in clinical practice", 2nd ed, 1997.

[5] S. Amir, C. Rabin and A. Galatzer, "Cognitive and behavioral determinants of compliance in diabetics", Health Soc. Work, Pp.144-151, 1990.

[6] The Diabetes Control and Complications Trial Research Group, “The effect of intensive treatment of diabetes on the development and progression of long-term complications in insulin-dependent diabetes mellitus", The New England Journal of Medicine, Vol. 329, Pp. 977-986, 1993.

[7] C.F. Amaral, M. Brischwein and B. Wolf, Multiparameter techniques for non-invasive measurement of blood glucose, Sensors and Actuators B: Chemical, Vol. 140, No. 1, Pp. 12-16, 2009.

[8] H.D. Park, K.J. Lee, H.R. Yoon and H.H. Nam, Design of a portable urine glucose monitoring system for health care. Computers in biology and medicine, Vol. 35, No. 4, Pp. 275-286, 2005.

[9] “Benedict's reagent”, en.wikipedia.org/wiki/Benedict's_reagent.

[10] B.D. Lewis, "Laboratory evaluation of the glucocard blood glucose test meter”, Clin. Chem. 38, Pp. 2093-2095, 1992. 